\title{
Analysis of risk factors for the development of a post-bronchoscopy respiratory infection in lung cancer patients
}

Masafumi Shimoda ( $\square$ yamigatari@outlook.jp)

Fukujuji Hospital https://orcid.org/0000-0002-2465-0118

Kazunari Yamana

Fukujuji Hospital

Ryozo Yano

Fukujuji Hospital

Masaoki Saitou

Fukujuji Hospital

Keiji Fujiwara

Fukujuji Hospital

Koji Furuuchi

Fukujuji Hospital

Takeshi Osawa

Fukujuji Hospital

Fumiko Uesugi

Fukujuji Hospital

Kenichi Arakawa

Fukujuji Hospital

Kozo Morimoto

Fukujuji Hospital

Yoshiaki Tanaka

Fukujuji Hospital

Hiroyuki Kokutou

Fukujuji Hospital

Masao Okumura

Fukujuji Hospital

Takashi Uchiyama

Fukujuji Hospital

Ken Ohta

Fukujuji Hospital

Kozo Yoshimori 


\section{Research article}

Keywords: bronchoscopy, respiratory infection, lung abscess, guide sheath, risk factor

Posted Date: November 26th, 2019

DOI: https://doi.org/10.21203/rs.2.17792/v1

License: (c) (i) This work is licensed under a Creative Commons Attribution 4.0 International License. Read Full License

Version of Record: A version of this preprint was published on October 12th, 2020. See the published version at https://doi.org/10.1016/j.jiac.2020.09.029. 


\section{Abstract}

Background: The development of pneumonia following bronchoscopy is very important as a postbronchoscopy complication. Most patients with post-bronchoscopy respiratory infections show typical pneumonia, and lung abscesses are rare. However, bronchoscopic techniques have advanced, and recently, we have observed patients with lung abscess after bronchoscopy. Therefore, the risk factors might vary from those in past reports. This study aims to identify the incidence of and risk factors for post-bronchoscopy respiratory infections. Methods: We retrospectively studied adult patients diagnosed with lung cancer by bronchoscopy at Fukujuji Hospital between January 2017 and June 2019. Patients in the infection and noninfection groups were compared. The incidence of lung abscess was compared between recent periods and 2013, when endobronchial ultrasonography with a guide sheath (EBUS-GS) was not yet used in our hospital. Results: We reviewed 327 patients, including 20 patients (6.1\%) in the infection group. The risk factors for infection were necrosis and/or a cavity in the tumor $(\mathrm{p}<0.001)$, large tumor diameter $(\geq 30 \mathrm{~mm})(p=0.003)$, and low serum albumin $(<4.0 \mathrm{~g} / \mathrm{dL})(p=0.012)$. We developed a predictive score that included these risk factors, and the area under the curve of the score was 0.737 ( $95 \%$ $\mathrm{Cl}$ : 0.610-0.864). Conversely, no significant differences in age, current smoking status, or abnormal bronchoscopic findings were observed, even though these factors were reported as risk factors in past reports. Other risk factors for infection were a high white blood cell count $(p=0.007)$, high C-reactive protein level $(p=0.014)$, and expression of programmed death-ligand 1 expression in the tumor cells $(p=0.033)$. In total, 12 patients had lung abscesses $(3.7 \%)$, which represents a higher incidence than that in 2013 (0.8\%). Other types of infection were post-obstructive pneumonia in four patients $(1.2 \%)$ and typical pneumonia in four patients (1.2\%). Conclusions: The risk factors for developing postbronchoscopy respiratory infection in our study varied from those in past reports, possibly because of the advancement of bronchoscopic techniques such as EBUS-GS.

\section{Introduction}

A flexible fiberoptic bronchoscope can safely perform assessments, aid in diagnosis, and provide treatment to patients with respiratory diseases [1], and complications associated with bronchoscopy occur in $0.2-5.6 \%$ of patients with a mortality rate of $0.004-0.02 \%$ [2-5]. The development of pneumonia following bronchoscopy is very important in patients suffering from lung cancer since pneumonia causes a delay in treating the malignancy [6]. Most of the patients with post-bronchoscopy respiratory infections show a new infiltrate or consolidation on chest radiographic findings similar to the signs of typical pneumonia $[3,6,7]$. Additionally, lung abscesses rarely develop in post-bronchoscopy respiratory infections and occurred in $0.22-1.06 \%$ of patients in a past report $[7,8]$. However, recently, we have observed patients with lung abscesses after bronchoscopy. Bronchoscopic techniques have been further developed to include methods such as endobronchial ultrasonography (EBUS), EBUS with a guide sheath (EBUS-GS) and EBUS-guided transbronchial needle aspiration (EBUS-TBNA); therefore, an updated review should be considered [8]. Some past reports have demonstrated the risk factors for post-bronchoscopy respiratory infection, such as an elderly age, current smoking status, and central location of the tumor [6, 
9]. However, the risk factors for post-bronchoscopy respiratory infection might differ from those in past reports. This study aims to identify the incidence of and risk factors for post-bronchoscopy respiratory infections.

\section{Methods}

\section{Study Design and Setting}

We retrospectively studied patients who were diagnosed with lung cancer by bronchoscopic examination at the Respiratory Disease Center of Fukujuji Hospital from January 2017 to June 2019. Patients with post-bronchoscopy respiratory infections were compared to patients who did not develop an infection after bronchoscopy. We selected adult patients (age $\geq 18$ years) with lung cancer who were diagnosed by a bronchoscopic examination. The main outcome of our study was the diagnosis of post-bronchoscopy respiratory infection. Data were collected about the symptoms, laboratory data, radiological findings, bronchoscopic findings, histopathologic findings, and other relevant findings. Patients who were diagnosed by EBUS-TBNA without transbronchial lung biopsy (TBLB) and those who could not be diagnosed with lung cancer by bronchoscopic examination were excluded. The types of postbronchoscopy respiratory infection in 2017-2019 were compared to those in 2013, when EBUS-GS was not yet used in our hospital. The study was approved by the Institutional Review Board of Fukujuji Hospital. Patient consent was not required. The decisions made by this board are based on and in accordance with the Declaration of Helsinki.

\section{Definitions}

Post-bronchoscopy respiratory infection was defined as a respiratory infection that developed a new or progressive infiltrate on chest radiographs or computed tomography (CT) scans with an elevated white blood cell count (WBC) and/or C-reactive protein (CRP) during the period from the bronchoscopic examination to starting treatment for lung cancer or within a month. The types of post-bronchoscopy infection are classified as lung abscesses in the tumor, post-obstructive pneumonia, and typical pneumonia (Fig. 1). Lung abscesses were defined as follows: (1) primary shadow developing enlargement, and (2) cavity formation, low-density area in a primary shadow appearing or enlargement in post-bronchoscopy respiratory infection. Post-obstructive pneumonia was defined as an infection of the lung parenchyma distal to a bronchial obstruction [10]. Typical pneumonia was defined as a new infiltrate or consolidation around the target lesion during bronchoscopic examination. Necrosis in the tumor was classified as a low-density area in the tumor with a maximum of 30 Hounsfield units nonenhanced CT scans $[11,12]$. Lung cancer staging referred to the $8^{\text {th }}$ edition of the TNM classification [13]. Abnormal bronchoscopic findings were identified with intrabronchial tumor observations via bronchoscopy.

\section{Statistical Methods}

All data were analyzed and processed using EZR, version 1.35 [14]. Student's t test, Mann-Whitney U test, and Fisher's exact test were used to compare groups. Sensitivity, specificity, and odds ratios were 
calculated. Receiver operating characteristic (ROC) curves and the area under the curve (AUC) were calculated for each predictor model. ROC curves determined the cutoff value, and the AUC accurately measured the prediction model. The level of statistical significance was set at $p=0.05$ (2-tailed).

\section{Results}

A total of 344 patients were diagnosed with lung cancer by bronchoscopic examinations at our hospital. Seventeen patients who were diagnosed with EBUS-TBNA without TBLB were excluded, so we reviewed 327 patients in our study. Among all patients, 20 patients (6.1\%) developed post-bronchoscopy respiratory infections (the infection group), and 307 patients were in the noninfection group (Table 1). The median age was 68.5 years old (IQR: 64.5-78.5), and there were 16 males (80\%) in the infection group. The infection group and the noninfection group were not significantly different in age (median (IQR): 68.5 years old (64.5-78.5) vs 73.0 years old (67.0-78.0), $p=0.453)$, sex (male: $\mathrm{n}=16(80.0 \%)$ vs $\mathrm{n}=181(59.0 \%), p=0.097)$, underlying diseases $(\mathrm{n}=16(88.9 \%)$ vs $\mathrm{n}=262(87.6 \%), p=0.165)$, current smoking status $(n=7(36.8 \% \%)$ vs $n=63(22.5 \%), p=0.165)$, and symptoms $(n=11(57.9 \%)$ vs $n=124$ $(40.8 \%), p=0.157)$. The histological classification of lung cancer showed almost no significant difference, and other cancer subtypes, including non-small cell carcinoma, carcinoid tumor, combined with some histologic types, and pleomorphic adenoma, were more common in the infection group than in the noninfection group $(\mathrm{n}=5$ (25.0\%) vs $\mathrm{n}=25(8.1 \%), p=0.027)$. Programmed death-ligand 1 (PD-L1) expression in the tumor cells was more common in the infection group than in the noninfection group ( $\mathrm{n}=$ $10(50.0 \%)$ vs $n=78(25.4 \%), p=0.033)$. In terms of lung cancer stage, compared to those in the noninfection group, patients in the infection group had higher stage disease, especially since no patients in the infection group had stage I disease. The laboratory findings before the bronchoscopic examination showed higher inflammation (median WBC (IQR): 7525 cells/ $\mu \mathrm{L}$ (6955-9155) vs $6660 \mathrm{cells} / \mu \mathrm{L}$ (53608190), $p=0.007$, median CRP (IQR): $0.59 \mathrm{mg} / \mathrm{dL}(0.16-2.71)$ vs $0.17(0.05-0.86), p=0.014)$ and lower serum albumin (median (IQR): $3.71 \mathrm{~g} / \mathrm{dL}(3.22-4.08)$ vs $4.12 \mathrm{~g} / \mathrm{dL}(3.80-4.33), p=0.012)$ in the infection group than in the noninfection group. In radiographic findings, patients in the infection group had larger tumor diameters (median (IQR): $41.3 \mathrm{~mm}$ (32.7-57.9) vs. $29.6 \mathrm{~mm}$ (18.2-46.3), $p=0.003$ ) and more necrosis and/or cavities in the tumors $(n=8(40.0 \%)$ vs. $n=29(9.4 \%), p<0.001)$ than those in the noninfection group. The incidence of using a guide sheath $(p=0.588)$, abnormal bronchoscopic findings $(p=0.175)$, and antibiotic prophylaxis $(p=0.429)$ were not significantly different between the two groups.

The odds ratios of a tumor diameter of $30 \mathrm{~mm}$ or more, necrosis and/or a cavity in the tumor, WBC of 6500 cells $/ \mu \mathrm{L}$ or more, CRP of $0.55 \mathrm{mg} / \mathrm{dL}$ or more, and serum albumin less than $4.0 \mathrm{~g} / \mathrm{dL}$ are shown in Table 2. Necrosis and/or a cavity in the tumor showed the highest odds ratio at 6.33 (95\% confidence level (Cl): 2.07-18.5, $p<0.001)$. We developed a predictive score for the development of post-bronchoscopy respiratory infections that included necrosis and/or cavities in the tumors, tumor diameter $\geq 30 \mathrm{~mm}$, and serum albumin $<4.0 \mathrm{~g} / \mathrm{dL}$ (Fig. 2). Each variable was assigned a value of 1 point, thus totaling 3 points. The ROC curve of the score demonstrated a high AUC of 0.737 (95\% Cl: $0.610-0.864)$. A score of 2 or more was regarded as the cutoff value, and the sensitivity, specificity, and odds ratio were $70.0 \%, 69.2 \%$, and 5.21 (95\% Cl: 1.81-17.1), respectively. 
Table 3 shows the characteristics of the infection group. Of all the patients, 12 patients had lung abscesses (3.7\%), 4 patients had post-obstructive pneumonia (1.2\%), and 4 patients had typical pneumonia (1.2\%). The median duration from the bronchoscopic examination to treatments for postbronchoscopy respiratory infection was 12.5 days (interquartile range (IQR): 7.0-18.3). Eight out of the 12 patients with lung abscesses showed necrosis and/or a cavity in the tumor; however, no patients with post-obstructive pneumonia and typical pneumonia showed those findings. All patients with postobstructive pneumonia showed abnormal bronchoscopic findings and underwent biopsy without EBUSGS. Conversely, many patients with lung abscesses and typical pneumonia underwent EBUS-GS and did not show abnormal bronchoscopic findings. One patient with a lung abscess required surgical treatment, and another patient died. In 2013, when EBUS-GS was not yet used in our hospital, 131 patients were diagnosed with lung cancer by bronchoscopic examination, and seven of these patients had postbronchoscopy respiratory infection (5.3\%), including only one patient with a lung abscess $(0.8 \%)$ and six patients with other types of pneumonia (4.6\%). The incidence of developing lung abscesses after bronchoscopy has increased in recent years (Fig. 3). In addition, the number of biopsies performed in 2019 was higher than that in $2017(p=0.018)$.

\section{Discussion}

This study identified the characteristics and risk factors for developing a post-bronchoscopy respiratory infection. A total of $6.1 \%$ of all patients developed post-bronchoscopy respiratory infections, and the risk factors were high WBC counts or CRP levels, low serum albumin levels, necrosis and/or a cavity in the tumor, and large tumor diameter. However, these risk factors differ from those in past reports $[6,9]$. We developed a predictive score that included necrosis and/or a cavity in the tumor, tumor diameter $\geq 30 \mathrm{~mm}$, and serum albumin $<4.0 \mathrm{~g} / \mathrm{dL}$. A score of a least 2 was a good predictor for developing a postbronchoscopy respiratory infection. In total, $60 \%$ of the patients with post-bronchoscopy respiratory infections showed lung abscesses. The incidence of developing lung abscesses after bronchoscopy has increased in recent years. We believe that these different risk factors are caused by the advancement of bronchoscopic techniques.

A past report from 2012 to 2013 suggested risk factors for post-bronchoscopy respiratory infection, such as an elderly age, current smoking status, and central location of the tumor [6]. However, these factors did not differ significantly between the infection group and the noninfection group in our study. These differences might be caused by the increasing incidence of lung abscesses after bronchoscopy since the bronchoscopic techniques have been changed. Generally, lung abscesses are rare in post-bronchoscopy respiratory infections and occur in $0.22-1.06 \%$ of the patients $[7,8]$. Similar to the percentage in past reports, $0.8 \%$ of the patients in our study had lung abscesses after bronchoscopy in 2013, when EBUS-GS was not yet used in our hospital. However, the incidence of lung abscesses after bronchoscopy increased to as high as $5.3 \%$ in 2019. Recently, the EBUS-GS technique has been used and has significantly increased the diagnosis rate of peripheral pulmonary lesions $[15,16]$. Moreover, the number of TBLB procedures performed is higher because the specimens from EBUS-GS are smaller in size than those from routine TBLB [15]. The British Thoracic Society guidelines for bronchoscopy recommend taking at least 
five biopsy samples [1], and an additional five bronchial forceps biopsies should be considered for phenotyping and genotyping exams [17]. In our hospital, the number of biopsy by bronchoscopy procedures performed increased from October 2018 because of phenotyping and genotyping, and the number of biopsies performed by bronchoscopy was significantly greater in 2019 than in 2017. Therefore, we thought that post-bronchoscopy lung abscesses can easily occur due to the type of approach, infection of the central tumor using EBUS-GS and increases in the number biopsies performed.

Lung abscesses are a type of liquefaction of the lung tissue and a formation of cavities containing necrotic debris or fluid caused by microbial infection [18]. Ishida $M$ et al. reported that a risk factor for post-bronchoscopy lung abscess was a tumor diameter of $30 \mathrm{~mm}$ or more [8], which is similar to our report. We demonstrated risk factors such as necrosis and/or a cavity in the tumor and large tumor diameter. Conversely, all patients with post-obstructive pneumonia showed abnormal bronchoscopic findings and did not show necrosis and/or cavities in the tumors. Patients with typical pneumonia after bronchoscopy did not show necrosis, a cavity in the tumor or abnormal bronchoscopic findings. Therefore, the risk factors for post-bronchoscopy respiratory infection might differ among types of infection.

Other risk factors for post-bronchoscopy respiratory infection were low serum albumin before the bronchoscopic examination and PD-L1 expression in tumor cells. Low serum albumin might indicate that the immune systems of the patients in the infection group were worse than those of the patients in the noninfection group. Generally, the nutritional status as assessed by the serum albumin level relates to the immune response, and malnutrition is a common cause of immunodeficiency $[19,20]$. In addition, PD-L1 expression in tumor cells is related to the immune system. The combination of programmed cell death 1 (PD-1) and PD-L1 leads to tumor immune evasion through the suppression of T cells [21], and the role of PD-1 is to regulate infections due to the limited function of macrophages as well as the T cellindependent B cell response [22]. A case report of a patient with a lung abscess after bronchoscopy showing high levels of PD-L1 was previously published [23]. Thus, immune disorder is an important risk factor for the development of post-bronchoscopy respiratory infections.

The British Thoracic Society guidelines for bronchoscopy do not recommend antibiotic prophylaxis before bronchoscopy for endocarditis, fever, or pneumonia [1]. In our study, antibiotic prophylaxis did not effectively prevent post-bronchoscopy respiratory infections. However, very few patients received antibiotic prophylaxis, so whether antibiotic prophylaxis was useful cannot be determined. Kanazawa $\mathrm{H}$ et al. reported that azithromycin was effective as prophylactic antibiotherapy in preventing infections post bronchoscopy compared to the no-treatment group ( $3.0 \%$ vs $14.8 \%, p=0.02)$ [7]. Generally, the common causative organisms of lung infections after bronchoscopy are oro- or nasopharyngeal microbes, such as Streptococcus, Staphylococcus, Moraxella, Neisseria, and anaerobic bacterial species [5]. Therefore, prophylactic antibiotherapy might be better adapted to anaerobic bacteria. Many patients with post-bronchoscopy respiratory infections canceled their planned oncologic treatments [6], and some post-bronchoscopy respiratory infections could not improve with antibiotics and required surgical 
invention [8]. Therefore, prophylactic antibiotherapy might be necessary for high-risk patients. A prospective study of effective antibiotic prophylaxis is required.

This investigation had several limitations. The study was conducted retrospectively in a single center. The target disease was lung cancer; therefore, the incidence in this study does not represent the overall incidence of bronchoscopy. Some medical data were not recorded. Some patients received prophylaxis antibiotherapy. We reviewed all types of infections together. Some patients did not have PD-L1 tumor cell expression data because the analysis was not requested or sample size from TBLB was too small.

\section{Conclusion}

The study demonstrated the characteristics of post-bronchoscopy respiratory infections, which was mainly lung abscesses. The risk factors were high inflammation, low serum albumin, necrosis and/or a cavity in the tumor, and large tumor diameter. In addition, we developed a good predictive score, which included necrosis and/or a cavity in the tumor, tumor diameter $\geq 30 \mathrm{~mm}$, and serum albumin $<4.0 \mathrm{~g} / \mathrm{dL}$. We believe that these risk factors vary from those in past reports because of the advancement of bronchoscopic techniques.

\section{List Of Abbreviations}

EBUS: endobronchial ultrasonography

EBUS-GS: endobronchial ultrasonography with a guide sheath

EBUS-TBNA: endobronchial ultrasonography-guided transbronchial needle aspiration

TBLB: transbronchial lung biopsy

CT: computed tomography

WBC: white blood cell count

CRP: C-reactive protein

ROC: receiver operating characteristic

AUC: area under the curve

IQR: interquartile range

PD-L1: programmed death-ligand 1

Cl: confidence interval

PD-1: programmed cell death 1 
EGFR: epidermal growth factor receptor

LDH: lactate dehydrogenase

\section{Declarations}

Ethics Approval and Consent to Participate:

The study was approved by the Institutional Review Board of Fukujuji Hospital. It was determined that patient consent was not required. The decisions made by this board are based on and in accordance with the Declaration of Helsinki.

Consent for Publication: Not applicable

Availability of Data and Materials:

The datasets used and/or analyzed during the current study are available from the corresponding author on reasonable request.

Competing Interests: All authors state that they have no conflicts of interest.

Funding: Not applicable

Authors' Contributions:

$K Y$ conceived and designed the work with the corresponding author. RY, MS, KF, KF, TO, FU, KA, KM, YT, $\mathrm{HK}, \mathrm{MO}, \mathrm{TU}, \mathrm{KO}$, and KY collected patient data. All authors read and approved the final manuscript.

Acknowledgments: Not applicable

\section{References}

1. Du Rand IA, Blaikley J, Booton R, Chaudhuri N, Gupta V, Khalid S, Mandal S, Martin J, Mills J, Navani $\mathrm{N}$, et al: British Thoracic Society guideline for diagnostic flexible bronchoscopy in adults: accredited by NICE. Thorax 2013, 68 Suppl 1:i1-i44.

2. Facciolongo N, Patelli M, Gasparini S, Lazzari Agli L, Salio M, Simonassi C, Del Prato B, Zanoni P: Incidence of complications in bronchoscopy. Multicentre prospective study of 20,986 bronchoscopies. Monaldi Arch Chest Dis 2009, 71:8-14.

3. Kanemoto K, Satoh H, Ishikawa H, Ishikawa S, Ohtsuka M, Sekizawa K: Prospective study of fever and pneumonia after flexible fiberoptic bronchoscopy in older people. J Am Geriatr Soc 2006, 54:827830.

4. Asano F, Aoe M, Ohsaki Y, Okada Y, Sasada S, Sato S, Suzuki E, Senba H, Fujino S, Ohmori K: Deaths and complications associated with respiratory endoscopy: a survey by the Japan Society for 
Respiratory Endoscopy in 2010. Respirology 2012, 17:478-485.

5. Stahl DL, Richard KM, Papadimos TJ: Complications of bronchoscopy: A concise synopsis. Int J Crit IIIn Inj Sci 2015, 5:189-195.

6. Takiguchi H, Hayama N, Oguma T, Harada K, Sato M, Horio Y, Tanaka J, Tomomatsu H, Tomomatsu $\mathrm{K}$, Takihara $\mathrm{T}$, et al: Post-bronchoscopy pneumonia in patients suffering from lung cancer: Development and validation of a risk prediction score. Respir Investig 2017, 55:212-218.

7. Kanazawa $\mathrm{H}$ : Efficacy of azithromycin administration in prevention of respiratory tract infection after bronchoscopic biopsy: a randomized, controlled trial. Respirology 2007, 12:70-75.

8. Ishida M, Shimazaki T, Suzuki M, Ariyoshi K, Morimoto K: Case series of lung abscesses following flexible bronchoscopy. Respir Investig 2015, 53:129-132.

9. Pereira W, Kovnat DM, Khan MA, lacovino JR, Spivack ML, Snider GL: Fever and pneumonia after flexible fiberoptic bronchoscopy. Am Rev Respir Dis 1975, 112:59-64.

10. Rolston KVI, Nesher L: Post-Obstructive Pneumonia in Patients with Cancer: A Review. Infect Dis Ther 2018, 7:29-38.

11. Yamashita K, Matsunobe S, Tsuda T, Okuda K, Matsumoto K, Oyanagi H, Konishi J: Intratumoral necrosis of lung carcinoma: a potential diagnostic pitfall in incremental dynamic computed tomography analysis of solitary pulmonary nodules? J Thorac Imaging 1997, 12:181-187.

12. Ye XD, Ye JD, Yuan Z, Dong S, Xiao XS: Characterization of solitary pulmonary nodules: Use of washout characteristics at contrast-enhanced computed tomography. Oncol Lett 2012, 3:672-676.

13. Goldstraw P, Chansky K, Crowley J, Rami-Porta R, Asamura H, Eberhardt WE, Nicholson AG, Groome P, Mitchell A, Bolejack V, et al: The IASLC Lung Cancer Staging Project: Proposals for Revision of the TNM Stage Groupings in the Forthcoming (Eighth) Edition of the TNM Classification for Lung Cancer. J Thorac Oncol 2016, 11:39-51.

14. Kanda Y: Investigation of the freely available easy-to-use software 'EZR' for medical statistics. Bone Marrow Transplant 2013, 48:452-458.

15. Zhang $\mathrm{L}, \mathrm{Wu} \mathrm{H}$, Wang $\mathrm{G}$ : Endobronchial ultrasonography using a guide sheath technique for diagnosis of peripheral pulmonary lesions. Endosc Ultrasound 2017, 6:292-299.

16. Tachihara M, Tamura D, Kiriu T, Tokunaga S, Hatakeyama Y, Shinke H, Nagano T, Nakata K, Hazeki N, Kamiryo H, et al: Bronchoscopy Using Virtual Navigation and Endobronchial Ultrasonography with a Guide Sheath (EBUS-GS) with or without Fluoroscopy for Peripheral Pulmonary Lesions. Kobe J Med Sci 2018, 63:E99-E104.

17. Dietel M, Bubendorf L, Dingemans AM, Dooms C, Elmberger G, Garcia RC, Kerr KM, Lim E, Lopez-Rios F, Thunnissen E, et al: Diagnostic procedures for non-small-cell lung cancer (NSCLC): recommendations of the European Expert Group. Thorax 2016, 71:177-184.

18. Kuhajda I, Zarogoulidis K, Tsirgogianni K, Tsavlis D, Kioumis I, Kosmidis C, Tsakiridis K, Mpakas A, Zarogoulidis $\mathrm{P}$, Zissimopoulos $\mathrm{A}$, et al: Lung abscess-etiology, diagnostic and treatment options. Ann Transl Med 2015, 3:183. 
19. Chandra RK: Nutrition and the immune system: an introduction. Am J Clin Nutr 1997, 66:460S-463S.

20. Chandra RK: Nutritional regulation of immunity and risk of infection in old age. Immunology 1989, 67:141-147.

21. Liu J, Zhong Y, Peng S, Zhou X, Gan X: Efficacy and safety of PD1/PDL1 blockades versus docetaxel in patients with pretreated advanced non-small-cell lung cancer: a meta-analysis. Onco Targets Ther 2018, 11:8623-8632.

22. Brown KE, Freeman GJ, Wherry EJ, Sharpe AH: Role of PD-1 in regulating acute infections. Curr Opin Immunol 2010, 22:397-401.

23. Migiyama Y, Anai M, Kashiwabara K, Tomita Y, Saeki S, Nakamura K, Okamoto S, Ichiyasu H, Fujii K, Kohrogi H: Lung abscess following bronchoscopy due to multidrug-resistant Capnocytophaga sputigena adjacent to lung cancer with high PD-L1 expression. J Infect Chemother 2018, 24:852-855.

\section{Tables}

Table 1. Baseline characteristics of the patients in the study 
Infection No infection $p$-value

$$
(\mathrm{n}=20) \quad(\mathrm{n}=307)
$$

Age, median (IQR), years

Sex (male/female)

Underlying disease, $\mathrm{n}(\%)^{\mathrm{a}}$

Current smoker, n (\%) ${ }^{\mathrm{b}}$

Experiencing symptoms, $\mathrm{n}(\%)^{\mathrm{C}}$

Histological classification of lung cancer

Adenocarcinoma, n (\%)

Squamous cell carcinoma, n (\%)

Neuroendocrine tumor, n (\%)

Other, n (\%)

EGFR mutation, n (\%)

PD-L1 expression, n (\%)

Stage

I, n (\%)

II, n (\%)

III, n (\%)

$\mathrm{IV}, \mathrm{n}(\%)$

Laboratory findings

WBC, median (IQR), cells $/ \mu \mathrm{L}^{\mathrm{d}}$

CRP, median (IQR), $\mathrm{mg} / \mathrm{dL}^{\mathrm{e}}$
$68.5(64.5-$

78.5)

$16 / 4$

16 (88.9)

7 (36.8)

11 (57.9)

9 (45.0)

6 (30.0)

$0(0)$

5 (25.0)

$3(15.0)$

$10(50.0)$

202 (65.8)

0.089

59 (19.2)

0.250

21 (6.8)

25 (8.1)

$68(22.1)$

78 (25.4)

0.033

0.628

0.027

0.583

$73.0(67.0-\quad 0.453$ 78.0)

$181 / 126$

0.097

1.000

0.165

0.157

$124(40.8)$ 
$\mathrm{LDH}$, median (IQR), U/L ${ }^{\mathrm{f}}$

Albumin, median (IQR), g/dL $\mathrm{dL}^{\mathrm{g}}$

$3.71(3.22-$

$$
\text { 4.08) }
$$

$4.12(3.80-$

Radiographic findings

Right lobe/left lobe

Upper lobe, n (\%)

Middle lobe/lingula, n (\%)

Lower lobe, n (\%)

Tumor diameter, median (IQR), cm

Necrosis and/or a cavity in the tumor, $\mathrm{n}(\%)$

Bronchoscopic technique

The number of biopsies performed, median (IQR)

Used a guide sheath, $\mathrm{n}(\%)$

Diagnosis by TBLB, n (\%)

Abnormal bronchoscopic findings

Fever ( $\geq 38$ degree C) within $24 \mathrm{hr}$ after bronchoscopy, n (\%)

Antibiotic prophylaxis, n (\%)
$9 / 11$

$10(50.0)$

$1(5.0)$

$9(45.0)$

41.3 (32.7-

57.9)

8 (40.0)
$178 / 128$

0.255

$142(46.3)$

0.821

$39(12.7)$

0.487

$122(39.7)$

0.815

$29.6(18.2-$

0.003 46.3)

$29(9.4)$ $<0.001$
$6.0(6.0-7.3) \quad 6.0(6.0-7.0) \quad 0.902$ $14(70.0) \quad 235(76.5) \quad 0.588$ 19 (95.0) $\quad 267(87.0) \quad 0.488$ $7(35.0) \quad 56(21.8) \quad 0.175$ $3(15.0) \quad 23(7.5) \quad 0.205$ $3(15.0)$
$29(9.4)$
0.429

a: infection group $n=18$, noninfection group $n=299$, b: infection group $n=19$, noninfection group $n=280$, c: infection group $n=19$, noninfection group $n=304$, d: infection group $n=20$, noninfection group $n=305$, e: infection group $n=20$, noninfection group $n=294$, $f$ : infection group $n=20$, noninfection group $n=297$, g: infection group $n=20$, noninfection group $n=298$.

IQR: interquartile range, EGFR: epidermal growth factor receptor, PD-L1: programmed death-ligand 1, TBLB: transbronchial lung biopsy, WBC: white blood cell count, CRP: C- 
Table 2. The odds ratios of several variables for diagnosing post-bronchoscopy respiratory infection

\begin{tabular}{|c|c|c|c|c|c|c|}
\hline & \multirow{2}{*}{$\begin{array}{l}\text { Odds } \\
\text { ratio }\end{array}$} & \multicolumn{2}{|c|}{$95 \% \mathrm{Cl}$} & \multirow[t]{2}{*}{ Sensitivity } & \multirow[t]{2}{*}{ Specificity } & \multirow{2}{*}{$\begin{array}{c}p- \\
\text { value }\end{array}$} \\
\hline & & $\begin{array}{l}\text { Lower } \\
\text { limit }\end{array}$ & $\begin{array}{l}\text { Upper } \\
\text { limit }\end{array}$ & & & \\
\hline Tumor diameter $\geq 30 \mathrm{~mm}$ & 4.01 & 1.25 & 16.9 & 80.0 & 50.2 & 0.010 \\
\hline $\begin{array}{l}\text { Necrosis and/or a cavity } \\
\text { in the tumor }\end{array}$ & 6.33 & 2.07 & 18.5 & 40.0 & 91.6 & $<0.001$ \\
\hline $\mathrm{WBC} \geq 6500$ & 4.79 & 1.35 & 26.0 & 85.0 & 45.9 & 0.009 \\
\hline $\mathrm{CRP} \geq 0.55$ & 2.94 & 1.07 & 8.35 & 55.0 & 70.7 & 0.023 \\
\hline Albumin $<4.0 \mathrm{~g} / \mathrm{dL}$ & 3.50 & 1.22 & 11.4 & 70.0 & 60.1 & 0.010 \\
\hline
\end{tabular}

Cl: confidence interval, WBC: white blood cell count, CRP: C-reactive protein

Table 3. The characteristics of the types of post-bronchoscopy respiratory infections

\begin{tabular}{lccc}
\hline & $\begin{array}{c}\text { Lung } \\
\text { abscess } \\
(\mathrm{n}=12)\end{array}$ & $\begin{array}{c}\text { Post-obstructive } \\
\text { pneumonia }\end{array}$ & $\begin{array}{c}\text { Typical pneumonia } \\
(\mathrm{n}=4)\end{array}$ \\
\hline $\begin{array}{l}\text { Necrosis and/or a cavity in the } \\
\text { tumor, n (\%) }\end{array}$ & $8(40.0)$ & $0(0.0)$ & $0(0.0)$ \\
Using a guide sheath, n (\%) & $10(70.0)$ & $0(0.0)$ & $4(100)$ \\
Abnormal bronchoscopic findings & $3(35.0)$ & $4(100)$ & $0(100)$
\end{tabular}

\section{Figures}




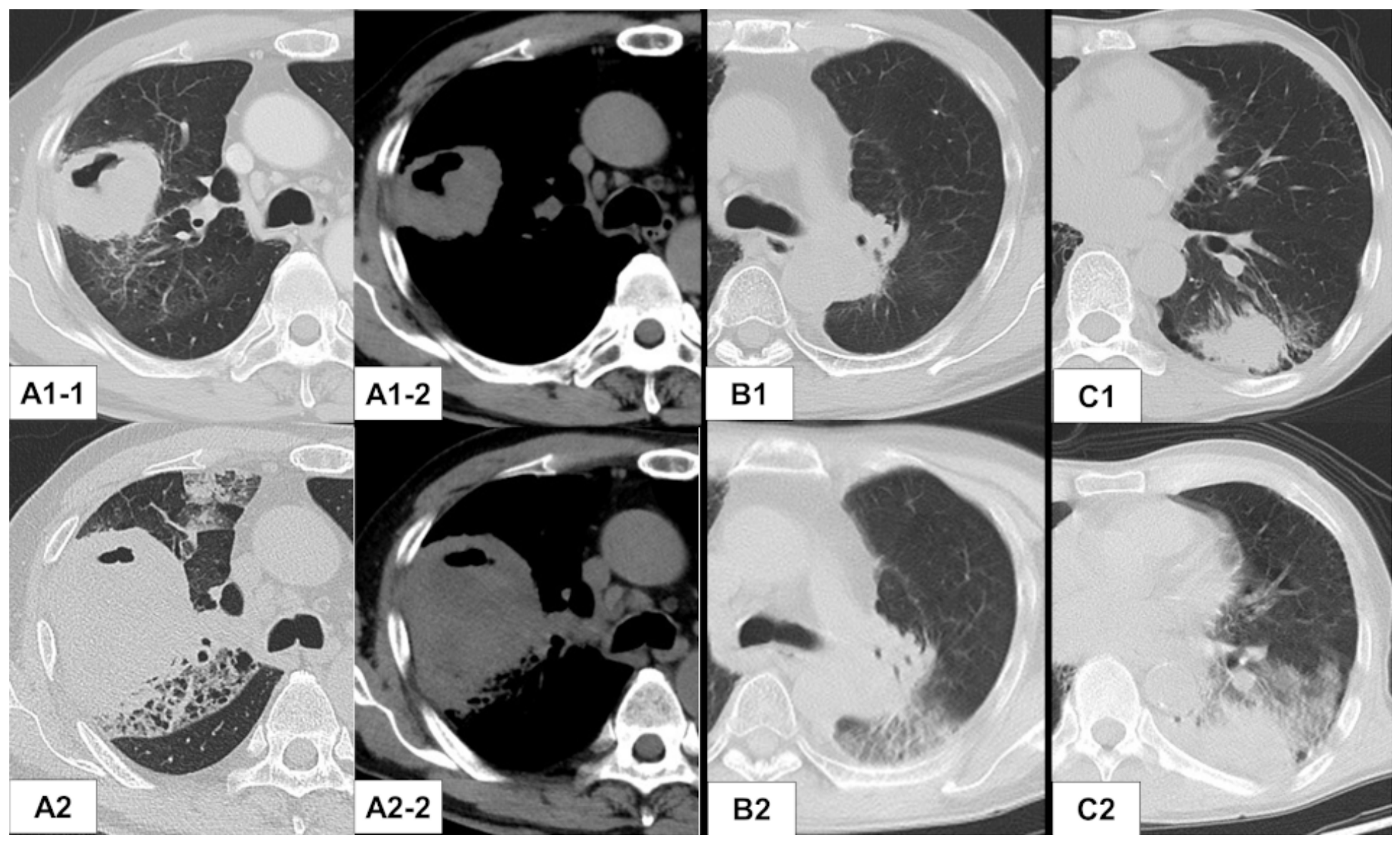

\section{Figure 1}

The types of post-bronchoscopy infections were classified as lung abscesses, post-obstructive pneumonia, and typical pneumonia A1-1: A tumor with central necrosis and a cavity A1-2: Mediastinal window setting of A1-1 A2-1: Lung abscess in a tumor after bronchoscopy A2-2: Mediastinal window setting of A2-2 B1: A tumor in the central area and obstructive bronchi B2: Post-obstructive pneumonia distal to a bronchial obstruction after bronchoscopy C1: A tumor in the lung field C2: A new infiltrate around the target lesion that appeared as pneumonia after bronchoscopy 


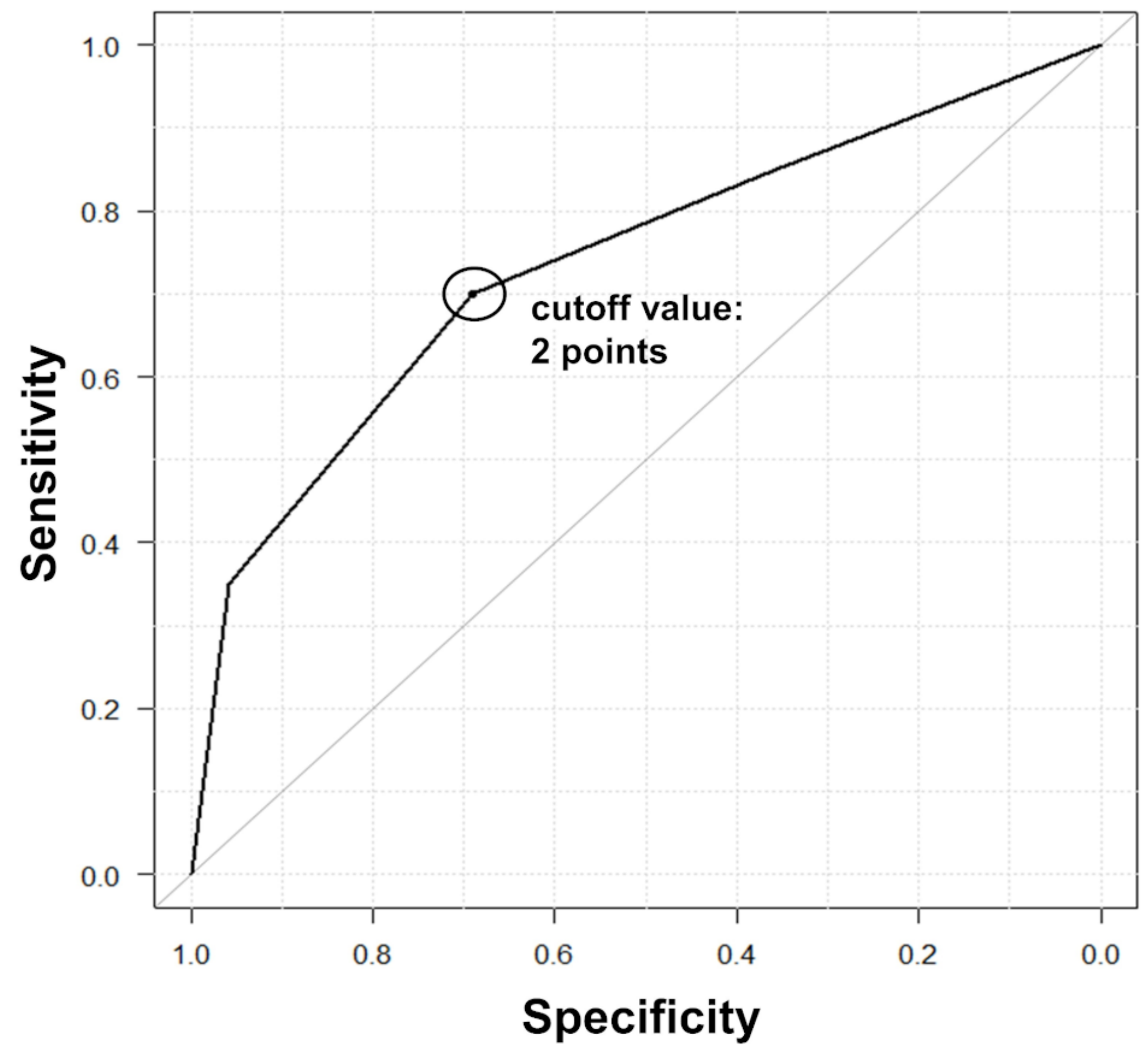

Figure 2

The receiver operating characteristic curve of the score for predicting post-bronchoscopy respiratory infections The area under the curve was 0.737 (95\% Cl: 0.610-0.864). The predictive accuracy for postbronchoscopy respiratory infection at a cutoff value of 2 points or more included a sensitivity of $70 \%$, specificity of $69.2 \%$, and odds ratio of 5.21 (95\% Cl: 1.81-17.1). Cl: confidence interval 


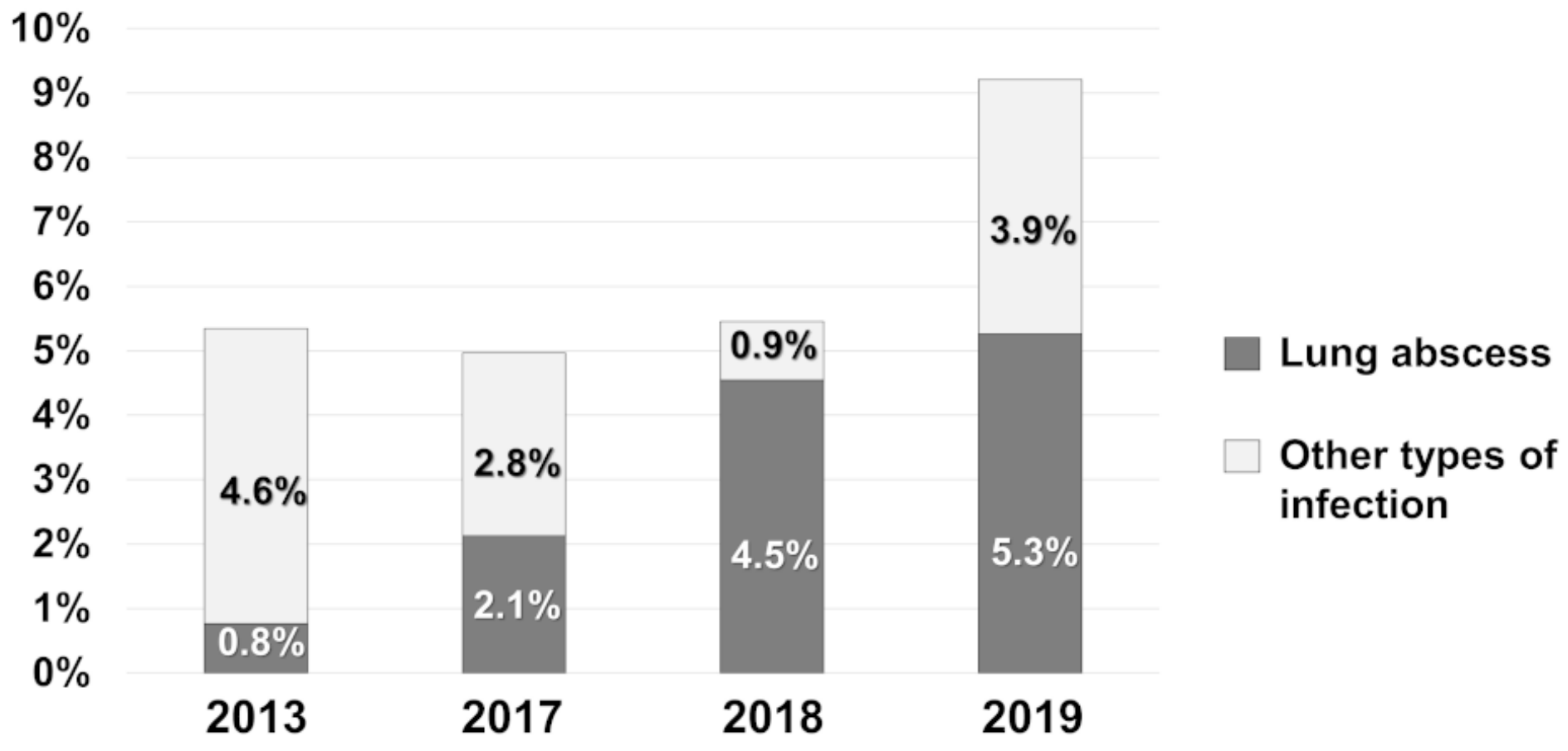

Figure 3

The incidence of developing post-bronchoscopy respiratory infections in 2013, 2017, 2018, and 2019 Data in 2019 were from January to June. 